\title{
Moderadores da Relação entre Congruência Pessoa-Ambiente e Satisfação Intrínseca no Trabalho
}

\author{
Fernanda de Souza Brito ${ }^{1}$ \\ Mauro de Oliveira Magalhães ${ }^{1}$ \\ ${ }^{1}$ Universidade Federal da Babia, Salvador, BA
}

\begin{abstract}
Resumo
A congruência pessoa-ambiente refere-se ao grau de similaridade entre características pessoais e ambientais. Este trabalho investigou moderadores da relação entre congruência pessoa-ambiente e satisfação intrínseca com o trabalho. Os moderadores investigados foram saliência de carreira, diferenciação entre interesses ocupacionais e tipos de interesse. Escalas de interesses e ambientes ocupacionais, satisfação intrínseca no trabalho e saliência de carreira foram respondidas por 842 trabalhadores brasileiros. A relação congruência-satisfação foram significativas somente em trabalhadores com baixa saliência de carreira, com elevada diferenciação de interesses e/ou que possuem interesse predominantemente investigativo. Conclui-se que, quanto menor a saliência de carreira do trabalhador e/ou mais diferenciado seu perfil de interesses, mais relevante é a congruência para a satisfação, especialmente em trabalhadores com interesse investigativo. Sugere-se que pessoas com elevada saliência de carreira são capazes de encontrar alternativas de realização profissional mesmo em ambientes de trabalho incongruentes com seus interesses.

Palavras-chave: congruência, satisfação no trabalho, interesses profissionais, desenvolvimento de carreira
\end{abstract}

Moderators of the Relationship Between Person-Environment Congruence and Intrinsic Job Satisfaction

\begin{abstract}
Personal-environmental congruence refers to the degree of similarity between personal and environmental characteristics. This work investigated moderators of the relationship between person-environment congruence and intrinsic satisfaction with work. The moderators investigated were career salience, differentiation between occupational interests, and interest types. Measures of interests and occupational environments, intrinsic job satisfaction, and career salience were answered by 842 Brazilian workers. The congruence-satisfaction relationship was significant only in workers with low career salience, with high interest differentiation and/or who have a predominantly investigative interest. We concluded that the lower the career salience of the worker and/ or more differentiated his/her profile of interests, the more relevant is the congruence to satisfaction, especially in workers with investigative interest. It is suggested that people with high career salience are able to find alternatives for professional achievement even in work environments that are incongruous with their interests.

Keywords: congruence; job satisfaction; professional interests; career development.
\end{abstract}

Moderadores de La Relación Entre Congruencia Persona-Ambiente y Satisfacción Laboral Intrínseca

\begin{abstract}
Resumen
La congruencia persona-ambiente se refiere al grado de semejanza entre características personales y ambientales. Este estudio investigó moderadores de relación entre congruencia persona-ambiente y satisfacción intrínseca en el trabajo. Los moderadores investigados fueron; relieves de la carrera, diferenciación entre intereses ocupacionales y tipo de intereses. Escalas de intereses y ambientes ocupacionales, satisfacción intrínseca en el trabajo y relieves de la carrera fueron respondidas por 842 trabajadores brasileños. La relación congruencia-satisfacción fue significativa solamente en trabajadores con bajo relieve de carrera, con elevada diferenciación de intereses y/o que poseen interés predominantemente investigativo. Se concluye que, cuánto menor el relieve de carrera del trabajador y/o más diferente su perfil de interés, más relevante es la congruencia para la satisfacción, sobre todo en los trabajadores con interés investigativo. Se sugiere que personas con elevado relieve de carrera son capaces de encontrar alternativas de realización profesional inclusive en ambientes de trabajo incongruentes con sus intereses.

Palabras-clave: congruencia; satisfacción en el trabajo; intereses profesionales; desarrollo de carrera
\end{abstract}

\section{Introdução}

A necessidade de compreender o ajuste do trabalhador ao seu ambiente ocupacional tem sido um tema relevante no campo da Psicologia Organizacional, em especial, nos estudos sobre carreira e gestão de pessoas (Kristof-Brown \& Billsberry, 2013). A premissa central desses estudos é que benefícios pessoais e organizacionais resultam do ajuste entre o trabalhador e sua ocupação. Kristof-Brown e Billsberry (2013) revisaram estudos sobre o ajustamento pessoa-ambiente e sinalizaram que, embora inúmeros aspectos pessoais e ambientais sejam investigados, predominam pesquisas que avaliam a similaridade entre interesses e ocupações, denominada de congruência.

O modelo de interesses e ambientes ocupacionais de Holland (1997) é considerado por muitos autores a perspectiva teórica mais compreensiva para se testar 
hipóteses relativas à congruência ( $\mathrm{Su}$, Murdock, \& Rounds, 2015; Wille, Tracey, Feys, \& De Fruyt, 2014), pois fornece descrições equivalentes de pessoas e ambientes, ou seja, ambos são categorizados em seis tipos: realista (R), investigativo (I), artístico (A), social $(\mathrm{S})$, empreendedor (E) e convencional (C) (modelo RIASEC). Tais tipos estão dispostos no formato de um hexágono, indicando o grau de afinidade entre eles. Tipos mais próximos no hexágono são considerados mais similares, compartilhando mais características (e.g., R e I; A e S), o que torna o perfil dos interesses e do ambiente mais consistente. Holland (1997) propôs que pessoas tendem a procurar ocupações que são congruentes com seus interesses e que o grau de congruência prediz resultados importantes, como bem-estar e satisfação no trabalho.

A congruência é avaliada a partir da comparação entre a classificação RIASEC dos interesses e dos ambientes ocupacionais. O perfil de interesses é obtido dos inventários de interesse embasados na teoria (Holland, 1997). Já, para a avaliação de ambientes ocupacionais, autores como Tinsley (2000) e Arnold (2004) recomendam medidas de autorrelato do trabalhador sobre as atividades e demandas de sua ocupação, pois são capazes de apreender especificidades ocupacionais que escapam a outros procedimentos. Após a classificação dos interesses e dos ambientes, os perfis são comparados para o cálculo do grau de congruência, que pode ser realizado por uma variedade de procedimentos. Grande parte dos índices de congruência avalia a distância hexagonal entre os tipos de interesses e de ambientes ocupacionais (Young, Tokar, \& Subich, 1998).

Nas pesquisas sobre congruência, uma variedade de consequentes tem sido investigada. Existem evidências de que as pessoas mudam de carreiras ou ocupações em busca de maior congruência (Donohue, 2006). No estudo de Rottinghaus, Hees e Conrath (2009), o nível de congruência diferenciou trabalhadores satisfeitos e insatisfeitos. Pseekos, Bullock-Yowell e Dahlen (2011) observaram que, quando um trabalhador percebe-se incongruente, há maior tendência a conflitos com os colegas de trabalho. A falta de congruência se mostrou um preditor importante de comportamentos contraproducentes no trabalho, que se destinam a prejudicar colegas e supervisores ou a organização como um todo, por meio do desperdício de materiais, por exemplo (Iliescu, Ispas, Sulea \& Ilie, 2014).

As pesquisas que examinam a relação entre congruência e variáveis relacionadas ao trabalho e à carreira têm sido sintetizadas em muitos estudos de revisão (Nauta, 2010; Nye, Su, Rounds, \& Drasgow, 2012; Spokane, Meir, \& Catalano, 2000; Tsabari, Tziner, \& Meir, 2005; Tranberg, Slane, \& Ekeberg, 1993). Dentre tais variáveis, este artigo destaca a satisfação no trabalho, que pode ser definida como o julgamento do trabalhador sobre o quanto o seu trabalho reforça positivamente seu comportamento (Dawis, 2005). Sobre as relações entre congruência e satisfação, os resultados têm sido contraditórios: alguns estudos apontam relações significativas positivas, indicando que quanto maior a congruência maior a satisfação no trabalho (e.g., De Fruyt, 2002), e outros não (e.g., Upperman \& Church, 1995). O estudo de metanálise de Tsabari et al. (2005) estima que a satisfação no trabalho apresenta um coeficiente de correlação de Pearson no valor de 0,17 com a congruência. É uma correlação baixa, mas é importante considerar que outras diferenças entre as pessoas podem estar moderando essa relação (Dik \& Hansen, 2011; Young et al., 1998; Tracey, Allen \& Robbins, 2012). Normalmente, uma variável moderadora é incluída no modelo quando há uma relação inconsistente ou fraca, não esperada, entre uma variável independente e outra dependente. Espera-se que o moderador influencie a direção e/ou força da relação entre essas variáveis. Em estudos correlacionais, um moderador é uma terceira variável que afeta a correlação entre duas outras variáveis (Baron \& Kenny, 1986; Vieira \& Faia, 2014).

\section{Moderadores da Relação entre Congruência e Satisfação}

Estudos que buscam identificar variáveis moderadoras da relação entre congruência e satisfação ainda são escassos (Dik \& Hansen, 2011; Tracey, 2007; Young et al., 1998). No entanto, é um campo de pesquisa promissor uma vez que busca compreender sob quais condições a congruência prediz satisfação. Por exemplo, Meir e Segal-Halevi (2001) observaram que a relação entre congruência e satisfação varia na dependência da importância atribuída pelo trabalhador ao seu grupo de trabalho. A elevada importância do grupo foi suficiente para altos níveis de satisfação, independentemente da congruência, ao passo que uma baixa importância do grupo esteve relacionada a baixos níveis de satisfação, exceto quando havia uma elevada congruência. Nos casos em que a importância do grupo esteve em um nível médio, quanto maior congruência, maior satisfação (Meir \& Segal-Halevi, 2001). Logo, a importância do grupo de trabalho foi um moderador importante da relação entre congruência e satisfação. 
Young et al. (1998) observaram que não houve correlação significativa entre congruência e satisfação em uma amostra de 483 trabalhadores americanos (da região centro-oeste dos EUA). Quando a amostra foi separada por tipos de interesse e de ambiente (RIASEC), os grupos revelaram correlações diferenciadas. As correlações congruência-satisfação mais fortes ocorreram em pessoas investigativas $(n=66 ; r=0,21$, $p<0,05)$ e em ambientes de trabalho investigativos $(n$ $=47 ; r=0,19, p<0,05)$. Os autores sugeriram que as preferências de pessoas com interesses predominantemente investigativos e respectivas funções de trabalho investigativas são bastante específicas e diferenciadas, e que essa elevada diferenciação de interesses e atividades resulta em maior importância da congruência para a vivência de satisfação no trabalho. Desse modo, recomendaram considerar o efeito moderador dos tipos RIASEC em pesquisas sobre a relação entre congruência e satisfação no trabalho.

A literatura nessa área tem sugerido ainda que a correlação entre congruência e satisfação pode ser maior entre pessoas que apresentem um perfil de interesses mais diferenciado, com maior clareza quanto a suas escolhas de carreira (Holland, 1997). A diferenciação de interesses na teoria de Holland refere-se ao grau com que a pessoa possui inclinações fortes para alguns tipos RIASEC e fracas para outros. A pesquisa sobre os efeitos da diferenciação de interesses enquanto moderadora da relação entre congruência e satisfação gerou resultados inconsistentes que levaram a uma redução de pesquisas sobre o tema (Nauta, 2010). Recentemente, Tracey, Wille, Durr e De Fruyt (2014) avaliaram a diferenciação de interesses da maneira tradicional proposta por Holland (1997), pela diferença entre o maior e o menor escore RIASEC, mas também pelo cálculo dos desvios dos escores RIASEC em relação à média, denominado de amplitude. Os autores constataram que as duas maneiras de avaliar a diferenciação de interesses apresentaram correlações significativas com a congruência. A amplitude obteve correlação mais forte e se mostrou moderadora da relação entre congruência e estabilidade ocupacional, indicando que quanto mais diferenciado é o perfil de interesses, mais a estabilidade ocupacional depende da congruência. Dada a escassez de pesquisas desse tipo, recomenda-se a investigação da diferenciação de interesses como moderador da relação entre congruência e satisfação.

Entre outras variáveis potencialmente moderadoras da relação entre congruência e satisfação, os construtos relacionados à centralidade ou à importância do trabalho, tais como a saliência de carreira, têm sido investigados. Essa categoria de construtos se reveste de importância na medida em que teorias de carreira têm sofrido duras críticas por implicitamente assumirem que a carreira é de importância central na vida de todas as pessoas (e.g., Worthington, Flores, \& Navarro, 2006). Essa pressuposição pode não ser válida para muitos (talvez a maioria) dos trabalhadores, considerando-se que preocupações de subsistência são prioritárias para uma proporção significativa da população mundial (Blustein, 2006).

Estudos iniciais sobre saliência de carreira, utilizando a medida proposta por Greenhaus (1971), destacaram diferenças entre homens e mulheres (Greenhaus, 1971; Illfelder, 1980; Moya, Expósito, \& Ruiz, 2000). Greenhaus (1971) constatou que a saliência de carreira estava relacionada ao grau de congruência pessoa-ocupação alcançado na escolha ocupacional somente em homens. Illfelder (1980) verificou que, em mulheres, quanto maior o medo do sucesso e a tipificação de papel sexual feminino, tanto menor a saliência de carreira. No estudo de Moya et al. (2000), não houve diferença de saliência de carreira entre homens e mulheres. Por outro lado, em mulheres, as variáveis relacionadas à saliência de carreira foram a realização educacional e a ideologia de igualdade de gênero; e, em homens, foram os atributos pessoais tipicamente masculinos: ambição, independência, força e ideologia sexista de gênero. Essas pesquisas sugerem que novos estudos sobre a saliência de carreira investiguem diferenças entre homens e mulheres.

O instrumento mais recente para avaliar a saliência de carreira é o Career Salience Questionnaire (CSQ), desenvolvido por Allen e Ortlep (2002). Os autores definiram saliência de carreira como a relativa proeminência e significado pessoal da carreira dentro do contexto mais amplo da vida de uma pessoa. Dik e Hansen (2011) utilizaram esse instrumento na investigação de possíveis efeitos moderadores da saliência de carreira, entre outras variáveis sobre a relação entre congruência e satisfação. Os resultados indicaram que a saliência de carreira não foi um moderador importante da relação entre congruência e satisfação. No entanto, a relação entre congruência e satisfação foi mais forte em trabalhadores que informaram menor envolvimento com o trabalho; que são menos motivados pelas recompensas intrínsecas ao desempenho; e que se percebem como menos influentes em seu ambiente de trabalho. Assim, em indivíduos que possuem menor envolvimento com o trabalho, a congruência é mais importante para a 
satisfação. Acredita-se que tais pessoas possam experimentar os inconvenientes da incongruência e os benefícios da congruência em maior intensidade.

Por outro lado, em trabalhadores altamente investidos em seus empregos e/ou que se percebem como influentes no seu local de trabalho, a relação entre congruência e satisfação não foi significativa, sugerindo que possam experimentar satisfação intrínseca com o trabalho independentemente do grau de congruência. Pode-se interpretar que essa independência resulte da capacidade dessas pessoas para modificar as condições do ambiente de modo a terem suas demandas satisfeitas. Assim, mesmo diante de ambientes com características incongruentes com seus interesses, profissionais com elevado envolvimento no trabalho estão dispostos a encontrar alternativas para terem seus interesses atendidos nesses mesmos ambientes (Dik \& Hansen, 2011). Desse modo, se o trabalho possui elevada importância, é provável que a pessoa desenvolva estratégias para se manter satisfeita, independentemente da congruência.

Por fim, foram encontrados somente dois estudos que avaliam a influência da saliência de carreira sobre a relação entre congruência e satisfação (Greenhaus, 1971; Dik \& Hansen, 2011), nenhum deles no Brasil, o que recomenda mais pesquisas sobre o tema. A compreensão da importância que os trabalhadores atribuem à carreira pode auxiliar no entendimento de comportamentos e atitudes relacionados ao seu ajuste ao ambiente ocupacional. Desse modo, sugere-se que a saliência de carreira, o nível de prioridade e cuidado dispensado ao desenvolvimento profissional pode oferecer novos insights para a investigação do ajuste pessoa-ambiente.

Em geral, nos estudos sobre a relação entre congruência e satisfação, esta última tem sido avaliada considerando diferentes aspectos do trabalho ou por um indicador global de satisfação (Rottinghaus et. al, 2009; Nauta, 2010). Ishitani (2010) recuperou o modelo pioneiro de Herzberg (1966) e defendeu a existência de dois tipos de satisfação no trabalho: extrínseca e intrínseca. A satisfação extrínseca envolve aspectos como salário, benefícios e suporte organizacional, que não estão teoricamente relacionados às predições teóricas do modelo RIASEC, pois independem da escolha ocupacional em si. Diante de condições de trabalho desfavoráveis, a insatisfação decorrente pode ser corrigida por meio de uma mudança de emprego, mas não de carreira ou área ocupacional. A satisfação intrínseca está relacionada ao conteúdo do trabalho e inclui aspectos como realização, importância do trabalho e crescimento pessoal. Ishitani (2010) constatou que a congruência foi mais efetiva para explicar a dimensão intrínseca da satisfação no trabalho do que a extrínseca.

Em síntese, este estudo analisou: a) relações entre satisfação intrínseca no trabalho e congruência pessoa -ambiente, considerando também análise da diferença de médias de satisfação entre grupos de baixa e alta saliência de carreira; b) o efeito moderador da saliência de carreira e da diferenciação de interesses na relação entre congruência e satisfação; c) o poder preditivo da congruência e demais variáveis investigadas sobre a satisfação intrínseca no trabalho, testando o modelo de regressão separadamente para homens e mulheres assim como em função dos tipos de interesses ocupacionais. No Brasil, não há registro de pesquisas com o uso de índices de congruência como preditores de atitudes e comportamentos no trabalho, o que destaca a relevância do presente estudo.

\section{Método}

\section{Participantes}

A amostra foi composta por 842 trabalhadores brasileiros (predominantemente baianos: 86\%) de diferentes áreas profissionais (majoritariamente em áreas administrativas e de prestação de serviços), com idades entre 25 e 76 anos $(M=39 ; D P=10), 329$ homens e 513 mulheres. O nível de escolaridade dos participantes variou do ensino fundamental $(1,9 \%)$ à pós-graduação $(33,1 \%)$, sendo que pouco mais da metade possui ensino superior $(50,1 \%)$. O tempo médio de trabalho na ocupação foi de oito anos.

\section{Instrumentos}

Escalas de Interesses Vocacionais - EIV (Teixeira, Castro, \& Cavalheiro, 2008). Desenvolvidas de acordo com o modelo de Holland (1997). Os itens são descrições de atividades que os participantes devem avaliar o quanto eles consideram interessantes, independentemente de suas habilidades para desempenhar a atividade descrita. Os participantes marcam suas respostas de acordo com uma escala tipo Likert de sete pontos (1 = Me desagrada muito; $7=$ Me agrada muito). A soma dos escores em cada dimensão indica os tipos de interesses predominantes do trabalhador. No presente estudo, os índices de consistência interna (alfa de Cronbach) das dimensões foram: $\mathrm{R}(\alpha=0,83)$, I $(\alpha=0,79), \mathrm{A}(\alpha=0,78), \mathrm{S}(\alpha=0,85), \mathrm{E}(\alpha=0,74)$ e $\mathrm{C}(\alpha=0,80)$.

Inventário de Classificação Ocupacional ICO (Brito \& Magalhães, 2017). Composto 37 itens 
descritores de atividades correspondentes às categorias do modelo RIASEC. O enunciado dos itens solicita que o participante marque a frequência com que as atividades descritas devem ser realizadas por uma pessoa na sua ocupação ou posto de trabalho atual. Os participantes marcaram suas respostas numa escala Likert de sete pontos $(1=$ Nunca; $7=$ Sempre $)$. A média dos itens em cada categoria RIASEC permite a classificação do tipo de ambiente de trabalho predominante da pessoa. As dimensões da escala, correspondentes às categorias RIASEC, apresentaram índices de consistência interna adequados (alfas de Cronbach): R $(\alpha=0,76), \mathrm{I}(\alpha=$ $0,85), \mathrm{A}(\alpha=0,88), \mathrm{S}(\alpha=0,86), \mathrm{E}(\alpha=0,80)$ e C $(\alpha=$ $0,79)$. Ademais, a análise fatorial indicou a adequação dos itens ao modelo de seis fatores, explicando $47,92 \%$ da variância total.

Escala de Satisfação Intrínseca com o Traba1ho. Foram desenvolvidos 10 itens com referência nos trabalhos de Quinn e Staines (1979) e Martins e Santos (2006). Essa escala buscou avaliar aspectos como realização e identificação com o trabalho, por meio de itens como: "Meu trabalho é interessante", "Eu me sinto emocionalmente ligado a esta área profissional". Portanto, refere-se à satisfação com aspectos do trabalho em si, em oposição à satisfação com recompensas extrínsecas do emprego. A resposta aos itens variou de acordo com uma escala tipo Likert de sete pontos (1 $=$ Discordo muito; $7=$ Concordo muito). Foi realizada uma análise dos principais eixos fatoriais da escala para testar sua unidimensionalidade e um único fator explicou $65,85 \%$ da variância (autovalor $>1$ ). Os itens obtiveram cargas fatoriais elevadas, variando de 0,70 a 0,88 . Ademais, a escala apresentou índice de consistência interna satisfatório (alfa de Cronbach $=0,95$ ).

Escala de Saliência de Carreira. É composta por seis itens, traduzidos e adaptados, neste estudo, do instrumento desenvolvido por Allen e Ortlepp (2002). A escala é introduzida com as seguintes instruções: "As frases a seguir foram projetadas para fazer você pensar sobre a importância de ter uma carreira em sua vida. Responda a estas frases em termos dos seus sentimentos sobre ter uma carreira pensada como uma trajetória de trabalho específica ou planejada para toda a sua vida, mais do que em termos dos seus empregos passados ou atuais" (Allen \& Ortlepp, 2002, p. 10). Os respondentes avaliaram o grau de concordância com cada uma das afirmativas de acordo com uma escala tipo Likert de sete pontos ( 1 = Discordo muito; 7 = Concordo muito $)$. $\mathrm{Na}$ presente amostra, uma análise dos principais eixos fatoriais revelou que um único fator explicou $45,3 \%$ da variância (autovalor $>1$ ). As cargas fatoriais dos itens variaram de 0,49 a 0,80 . O alfa de Cronbach foi 0,82 .

\section{Procedimentos}

A coleta de dados aconteceu entre os anos de 2015 e 2016. A aplicação do instrumento ocorreu por conveniência nos formatos digital (Plataforma Google Docs) e impresso. O link foi divulgado por e-mail e em redes sociais. A coleta presencial foi realizada pelos pesquisadores e estudantes de graduação em Psicologia vinculados ao grupo de pesquisa mediante visitas a associações de trabalhadores aposentados. As determinações éticas da resolução 196/1996 do Conselho Nacional de Saúde sobre a pesquisa com seres humanos foram atendidas, com aprovação do Comitê de ética em Pesquisa do Instituto de Psicologia da UFBA.

\section{Análise de Dados}

A análise de dados foi realizada por meio do Statistical Package for the Social Sciences - SPSS (versão 21.0). O índice de congruência foi calculado com os escores obtidos pelos participantes no ICO e nas EIV. O índice de congruência utilizado foi proposto no presente estudo e nomeado "C-tipos". O C-tipos foi obtido a partir dos seguintes passos: a) os escores dos três tipos adjacentes no hexágono foram somados (e.g., $\mathrm{R}+\mathrm{I}+\mathrm{A}$; $\mathrm{I}+\mathrm{A}+\mathrm{S}$, etc.), resultando em seis trios (RIA, IAS, ASE, SEC, ECR, CRI); b) dentre esses seis trios, o trio que possuía maior escore era classificado como predominante nos perfis de interesses e de ambientes ocupacionais; c) os trios predominantes de interesse e de ambiente eram comparados. Desse modo, o cálculo da congruência foi feito com base na comparação entre trios de tipos adjacentes dos interesses e dos ambientes, resultando num índice que variou de 3 a 0 . O valor 3 foi atribuído quando o trio de letras do interesse e do ambiente eram idênticas, 2 quando o trio de letras compartilhavam duas letras em comum (e.g., interesse RIA com ambiente IAS ou CRI), 1 quando o trio de letras compartilhavam uma letra em comum (e.g., interesse RIA com ambiente ASE ou ECR) e, por fim, 0 quando o trio de letras estavam em posições opostas (e.g., interesse RIA com ambiente SEC).

Ainda utilizando as Escalas de Interesses Vocacionais, foram calculados os escores de diferenciação, de acordo com a recomendação de Holland (1997), que considera que quanto maior a distância entre o escore do maior interesse e do menor interesse da pessoa, mais diferenciado será seu perfil. Logo a diferenciação foi 
obtida pela subtração do maior escore e menor escore de interesse vocacional.

Após o cálculo da congruência e da diferenciação, a análise de dados foi realizada em três etapas: a) foram realizadas análises de correlação entre as variáveis investigadas; b) as hipóteses de moderação foram testadas seguindo dois procedimentos descritos a seguir; c) por fim, a análise dos dados buscou explorar as variáveis investigadas como preditoras da satisfação intrínseca por meio de análises de regressão. No que se refere ao teste das hipóteses de moderação, no primeiro procedimento a amostra foi separada por níveis de alta e baixa saliência de carreira, alta e baixa diferenciação de interesses e classificada por tipos RIASEC de interesse. Foram realizadas análises da correlação entre congruência e satisfação para cada um desses níveis. Quando há correlação significativa em uma das condições e não em outra, existe moderação.

No segundo procedimento, as hipóteses de moderação foram testadas por meio de um modelo de regressão obtido por meio do macro PROCESS para SPSS e SAS (Hayes, 2012-2018), composto pela variável preditora (congruência), pelas moderadoras (saliência de carreira; diferenciação) e também pela interação entre elas, uma variável interativa (mensurada pela multiplicação entre preditor e moderador). De acordo com esse procedimento, há moderação quando o coeficiente de regressão da variável interativa (beta) é significativamente diferente de zero (Baron \& Kenny, 1986; Vieira \& Faia, 2014).

\section{Resultados e Discussão}

\section{Análises de Correlação e Moderação}

Inicialmente foram conduzidas análises de correlação entre as variáveis, considerando a amostra total $(N=842)$. Em seguida, as análises de correlação foram utilizadas para testar as hipóteses de moderação. Por fim, as variáveis investigadas foram analisadas como preditores da satisfação intrínseca. No que se refere às análises de correlação, a satisfação intrínseca com o trabalho apresentou correlação positiva e significativa com a congruência $(r=0,20, p<0,01)$. A correlação entre satisfação e saliência de carreira foi de $0,37(\phi<0,01)$. $O$ índice de congruência não apresentou correlação com saliência de carreira, nem com a diferenciação de interesses para a amostra total. De acordo com Baron e Kenny (1986), para testar a hipótese de moderação, é desejável que a variável moderadora (saliência de carreira; diferenciação) não esteja relacionada à variável preditora (congruência).

Para explorar efeitos moderadores, a amostra foi dividida por níveis de alta e baixa saliência de carreira, alta e baixa diferenciação e ainda por tipos de interesse predominante, refazendo as correlações congruênciasatisfação. No que diz respeito à saliência de carreira, a amostra foi dividida por níveis: 1) alta saliência, pessoas que tiveram escores maiores que a soma da média com o desvio padrão; e 2) baixa saliência, pessoas com escores menores que a subtração do desvio padrão $(D P)$ da média $(M)$. Em trabalhadores com alta saliência $(n=139)$, a correlação congruência-satisfação não foi significativa $(r=0,14, p=0,10)$. Ao passo que em trabalhadores com baixa saliência $(n=141)$ a correlação congruência-satisfação foi $r=0,30(p<0,01)$.

A diferença entre as médias de satisfação intrínseca com o trabalho entre os grupos de alta saliência $(M=58 ; D P=15,4)$ e baixa saliência de carreira $(M$ $=43 ; D P=15,7)$ foi significativa $[t(278)=8,1, p<$ $0,01]$. Pode-se concluir que as pessoas com elevada saliência de carreira estão significativamente mais satisfeitas com aspectos intrínsecos do seu trabalho do que pessoas com baixa saliência de carreira. No entanto, pessoas que atribuem menor importância às suas carreiras estão mais suscetíveis aos efeitos da congruência na satisfação, indicando que quanto menor a saliência, mais importante se torna desempenhar uma ocupação congruente.

É possível que pessoas com elevada saliência de carreira, encontrem uma forma de se ajustar a ambientes incongruentes, pois provavelmente possuem metas de longo prazo, fazendo com que elas percebam compensações futuras para os esforços atuais. Logo, para elas a congruência parece não influenciar sua satisfação, pois mesmo exercendo uma ocupação incongruente com seus interesses, sua satisfação pode advir da sensação de caminhar em direção a objetivos de longo prazo. Nesses casos, a saliência de carreira parece amortecer ou atenuar os efeitos da incongruência sobre a satisfação.

Após a análise das correlações entre congruência e satisfação entre os grupos de alta e baixa saliência de carreira, foi realizada a análise do efeito moderador da saliência de carreira por meio do modelo de regressão que inclui a variável interativa (multiplicação entre congruência e saliência). Nesse caso, a variável interativa não apresentou poder de explicação direta para a satisfação intrínseca (Beta $=-2,21, p>0,23$, não significativo). Quando o coeficiente de regressão da variável 
de interação não é significativo, a hipótese de moderação não se confirma, indicando que a saliência não modera a relação entre congruência e satisfação. Trata-se de uma primeira pesquisa brasileira, portanto tal resultado contraditório revela a necessidade de mais estudos sobre o tema.

Explorou-se também o efeito moderador da diferenciação de interesses na relação congruênciasatisfação, para testar a hipótese de Holland (1997) de que essa relação seria mais forte em trabalhadores com um perfil de interesses mais diferenciado. A amostra total foi dividida em trabalhadores com alta $(n$ $=163)$ e baixa diferenciação $(n=163)$. A correlação congruência-satisfação foi significativa somente em trabalhadores com interesses altamente diferenciados $(r=0,42, p<0,01)$. Em trabalhadores com interesses pouco diferenciados a correlação não foi significativa $(r$ $=0,09, p=0,23)$. Desse modo, a elevada diferenciação de interesses torna o trabalhador mais vulnerável para vivenciar consequências da congruência na satisfação. Quando o efeito moderador da diferenciação de interesses foi testado a partir da análise de moderação que inclui a variável de interação (Beta $=5,65, p>0,00)$, a diferenciação se confirma como moderadora da relação entre congruência e satisfação.

Para explorar os efeitos dos tipos de interesse na relação congruência-satisfação, os trabalhadores foram agrupados em função do tipo de interesse predominante. As correlações entre congruência e satisfação foram significativas somente para os tipos I, A e S: Investigativo $(n=102, r=0,38, p<0,01)$, Artístico $(n=46, r=0,42, p<0,01)$ e Social $(n=433, r=$ $0,18, p<0,01)$. As correlações não foram significativas para os tipos R, E e C: Realistas $(n=74, r=-0,09, p$ $=0,44)$, Empreendedores $(n=121, r=0,12, p=0,18)$ e Convencionais $(n=66, r=0,25, p=0,04)$. Esse resultado se repete mesmo quando se realiza uma correlação parcial, controlando os efeitos da saliência de carreira e da diferenciação de interesses na correlação congruência-satisfação. As pesquisas sobre as características de personalidade dos tipos RIASEC revelaram que indivíduos dos tipos I, A, e S tendem a apresentar uma menor conformidade e deferência a figuras de autoridade, a serem mais questionadores, autônomos e a valorizar a liberdade de expressão, em comparação a tipos empreendedores, convencionais e realistas (Holland, 1997; Hogan \& Blake, 1999). Tais características podem explicar o fato de que a satisfação para os tipos I, A e S seja mais dependente do exercício de uma ocupação congruente com seus interesses, uma vez que para eles é mais difícil adaptar-se ou conformar-se a uma realidade ocupacional que não atenda às suas expectativas.

Pesquisas sobre carreira têm apontado que trabalhadores com perfil tipicamente investigativo tendem a possuir maior identidade vocacional (Leong \& Morris, 1989) e maior entrincheiramento de carreira (Magalhães \& Gomes, 2007). Os autores concluíram que tipos investigativos são mais identificados com a atividade de trabalho em si, apresentando apego emocional às especificidades da tarefa. Desse modo a congruência tem maior poder preditivo sobre a satisfação intrínseca no trabalho em tipos investigativos, principalmente quando comparados a pessoas com interesses empreendedores, pois as últimas são descritas como preocupadas com poder e ganhos financeiros, valorizando aspectos extrínsecos da atividade profissional (salário, benefícios, oportunidades de reconhecimento) (Amabile, Hill, Hennessey, \& Tighe, 1994). Nesse sentido, Wille et. al. (2014) constataram que, embora os interesses ocupacionais sejam relativamente estáveis ao longo do tempo, pessoas empreendedoras são mais propensas a mudanças em seus interesses, comparativamente aos demais.

\section{Preditores da Satisfação Intrínseca no Trabalho}

Foram conduzidas análises de regressão que avaliaram o poder preditivo das variáveis investigadas sobre a satisfação intrínseca com o trabalho. A análise de regressão foi realizada por meio do método stepwise, tendo como variável dependente a satisfação intrínseca no trabalho e como variáveis independentes congruência, saliência de carreira, idade e escolaridade. O método stepwise é recomendado em estudos exploratórios, cuja a seleção de entrada dos preditores na equação é feita estatisticamente (Abbad \& Torres, 2002). As variáveis independentes juntas explicaram 19,5\% da variância da satisfação intrínseca com o trabalho. No primeiro passo da análise, a saliência de carreira explicou 13,6\% da variância da satisfação. Incorporando a congruência (C-tipos) no modelo, houve um incremento de 3,3\% na variância explicada da satisfação. Por fim, a idade e a escolaridade contribuíram com $1,9 \%$ e $0,7 \%$ respectivamente.

Não houve diferenças relacionadas ao sexo em saliência de carreira, congruência e satisfação no trabalho. Por outro lado, a análise dos preditores da satisfação intrínseca revelou padrões distintos em homens $(n=$ $329)$ e mulheres $(n=513)$. Em homens a variância explicada foi de $24,8 \%$ e em mulheres foi de $18,4 \%$, logo, reduzida em 6,4\% comparativamente aos homens. Em 
comparação às mulheres, a satisfação intrínseca com o trabalho para os homens depende mais da saliência de carreira, da similaridade de seus interesses com a ocupação desempenhada (congruência) e da idade.

As mulheres brasileiras, comparativamente aos homens, possuem mais dificuldade para ocupar posições de trabalho qualificadas e com remuneração compatível (Cavallini, 2018; Rolim, 2018). É possível cogitar que a satisfação com o trabalho em mulheres esteja mais associada à obtenção de reconhecimento e remuneração adequados do que realizar atividades congruentes. Assim, sugere-se que mulheres possuam maior tolerância a aspectos incongruentes do seu ambiente de trabalho, sendo a congruência menos importante para a sua satisfação.

Nesse sentido, o nível de escolaridade foi um preditor da satisfação intrínseca com o trabalho para as mulheres e não para os homens. Moya et al. (2000) observou que a escolaridade que foi preditor da saliência de carreira somente em mulheres. Esses resultados sugerem que as mulheres percebem suas conquistas educacionais como um critério importante para definir a saliência de carreira e a satisfação no trabalho.

Além da separação da amostra por sexo, análises de regressão foram conduzidas agrupando os trabalhadores por tipo de interesse ocupacional predominante. A saliência de carreira foi o único preditor da satisfação em trabalhadores classificados como realistas
(26,4\%), artísticos (40,3\%), empreendedores $(21,5 \%)$ e convencionais $(18,9 \%)$. Entre outros preditores, nos tipos investigativos contribuiu com $26,2 \%$ da variância de satisfação e em tipos sociais com 5,2\%. É possível supor que indivíduos que posicionam o papel de trabalho como prioritário em suas vidas tendem a persistir e avançar em suas metas de carreira, obtendo assim maior satisfação no trabalho.

Em trabalhadores com interesses predominantemente artísticos a saliência de carreira mostrou ter uma influência importante no quanto o indivíduo obtém satisfação intrínseca do seu trabalho. A atividade artística caracteriza-se por demandar soluções únicas e criativas que requerem um elevado envolvimento pessoal do trabalhador (Holland, 1997). Nesse sentido, pode-se dizer que são atividades que proporcionam um grau elevado de satisfação intrínseca. Assim, a elevada saliência de carreira nessa área de trabalho pode favorecer mais a satisfação intrínseca do que a satisfação com aspectos extrínsecos à tarefa (remuneração etc.). Nesse sentido, sabe-se das dificuldades associadas ao desenvolvimento de uma carreira artística e da necessidade de elevado investimento pessoal para que esta seja bem-sucedida em aspectos, tais como remuneração e benefícios.

Em tipos sociais os preditores de satisfação foram saliência de carreira (Beta $=0,235, p<0,01)$, escolaridade $($ Beta $=0,171, p<0,01)$, idade $($ Beta $=0,169, p$

Tabela 1.

Preditores de Satisfação Intrínseca com o Trabalho entre Homens e Mulheres

\begin{tabular}{|c|c|c|c|c|c|c|c|}
\hline \multirow{3}{*}{ Variáveis } & \multicolumn{3}{|c|}{ Homens $(n=329)$} & \multicolumn{4}{|c|}{ Mulheres $(n=513)$} \\
\hline & \multicolumn{3}{|c|}{ Passos } & \multicolumn{4}{|c|}{ Passos } \\
\hline & 1 & 2 & 3 & 1 & 2 & 3 & 4 \\
\hline \multirow[t]{2}{*}{ Saliência de carreira } & 0,446 & 0,439 & 0,436 & 0,329 & 0,318 & 0,298 & 0,311 \\
\hline & $(0,000)$ & $(0,000)$ & $(0,000)$ & $(0,000)$ & $(0,000)$ & $(0,000)$ & $(0,000)$ \\
\hline \multirow[t]{2}{*}{ Congruência C-tipos } & & 0,169 & 0,165 & & 0,192 & 0,187 & 0,189 \\
\hline & & $(0,001)$ & $(0,001)$ & & $(.000)$ & $(.000)$ & $(0,000)$ \\
\hline \multirow[t]{2}{*}{ Idade } & & & 0,145 & & & 0,153 & 0,129 \\
\hline & & & $(0,003)$ & & & & $(0,001)$ \\
\hline \multirow[t]{2}{*}{ Escolaridade } & & & & & & & 0,152 \\
\hline & & & & & & & $(0,000)$ \\
\hline $\mathrm{R} 2$ & 0,199 & 0,227 & 0,248 & 0,108 & 0,145 & 0,168 & 0,184 \\
\hline$\Delta \mathrm{R} 2$ & - & 0,028 & 0,021 & - & 0,037 & 0,023 & 0,016 \\
\hline $\mathrm{F}$ & 81,102 & 47,927 & 35,793 & 61,895 & 43,175 & 34,214 & 28,672 \\
\hline
\end{tabular}


$<0,01)$ e congruência (Beta $=0,162, p<0,01)$; explicando $13,6 \%$ da variância. A congruência contribuiu com 2,5\% de explicação da variância total. Destaca-se nos tipos investigativos a maior proporção de variância de satisfação no trabalho explicada pela congruência (7,9\%) além da saliência de carreira. Nesse grupo, a saliência de carreira, a congruência e a diferenciação de interesses explicaram 37\% da variância da satisfação intrínseca (Tabela 2). Esses dados sugerem que a congruência é especialmente importante para a satisfação em trabalhadores de interesses investigativos.

Ainda em tipos investigativos, observou-se que a diferenciação de interesses foi um preditor negativo da satisfação intrínseca com o trabalho. Assim, quanto menos diferenciado o perfil de interesses desses trabalhadores, maior sua satisfação intrínseca com o trabalho. Possivelmente, a baixa diferenciação dos interesses em trabalhadores investigativos facilita o ajustamento a contexto de trabalhos diversos do ideal investigativo, favorecendo a congruência e satisfação com o trabalho. Associado a isso, e também à observada importância da congruência para a satisfação desses profissionais, Magalhães e Gomes (2007) relacionaram a identidade de carreira e o alto grau de especialização profissional dos tipos investigativos a uma maior tendência ao entrincheiramento de carreira quando comparados a tipos empreendedores e realistas. Esse entrincheiramento revela a dificuldade desses indivíduos para cogitar e aceitar alternativas às suas atividades atuais de trabalho, dada a sua identificação com elas e o alto grau de especialização associado.

\section{Considerações Finais}

Este estudo investigou relações entre congruência e satisfação intrínseca com o trabalho e explorou variáveis moderadoras dessa relação. Além disso, foi verificado o poder preditivo das variáveis investigadas sobre a satisfação intrínseca, analisando tanto a amostra total quanto dividida entre trabalhadores do sexo masculino e do sexo feminino e entre trabalhadores com tipologias de interesses diferenciadas. A intensidade da relação entre congruência e satisfação, neste estudo, foi um pouco superior ao estudo de metanálise mais recente de Tsabari et al. (2005) mas similar aos estudos de revisão anteriores (e.g., Tranberg et al., 1993).

É importante destacar dois aspectos inovadores deste estudo: (a) a utilização de medida de autorrelato para avaliação dos ambientes ocupacionais; e (b) a criação de um novo índice de congruência (C-tipos). A medida de autorrelato foi o Inventário de Classificação Ocupacional - ICO, que permitiu verificar o tipo de ambiente de acordo com o modelo hexagonal de Holland (1997). Considera-se que o ICO demonstrou utilidade para avaliação da congruência pessoa-ambiente e recomenda-se que pesquisas futuras possam recorrer a medidas desse tipo, pois leva em conta a avaliação do trabalhador sobre seu próprio contexto ocupacional. Naturalmente, admite-se que o trabalhador seja melhor fonte de informações das especificidades do seu ambiente ocupacional do que amplas categorias ocupacionais descritas em dicionários de ocupações (Tinsley, 2000; Arnold, 2004). A partir do ICO, foi gerado o

Tabela 2.

Preditores de Satisfação Intrínseca com o Trabalho entre Trabalhadores Predominantemente Investigativos $(n=102)$

\begin{tabular}{lccc}
\hline & & Passos & \\
\hline Variáveis & 1 & 2 & 3 \\
Saliência de carreira & 0,520 & 0,490 & 0,468 \\
& $(0,000)$ & $(0,000)$ & $(0,000)$ \\
Congruência - C-tipos & & 0,293 & 0,316 \\
& & $(0,001)$ & $(0,000)$ \\
Diferenciação & & & $-0,190$ \\
& & & $(0,022)$ \\
R2 & 0,262 & 0,341 & \\
$\Delta$ R2 & - & 0,079 & 0,370 \\
F & 35,15 & 25,84 & 0,029 \\
\hline
\end{tabular}


C-Tipos, índice de congruência que comparou trios de tipos adjacentes de interesses e ambientes ocupacionais RIASEC. O C-tipos apresentou uma correlação mais elevada com satisfação, comparado às correlações apresentadas em estudos de revisão (Tsabari et al., 2005), o que indica sua adequação para fins de avaliação da congruência pessoa-ambiente.

A principal contribuição deste estudo refere-se à investigação de variáveis moderadoras da relação entre congruência e satisfação. Pesquisas desse tipo ainda são escassas na literatura, embora sejam consideradas promissoras de novas contribuições (Tracey, 2007; Dik \& Hansen, 2011). Neste estudo, a correlação congruênciasatisfação foi mais forte em trabalhadores com baixa saliência de carreira, com elevada diferenciação em seus perfis de interesse e com perfis predominantemente investigativos, artísticos ou sociais.

Pode-se concluir que quando o trabalhador atribui pouca importância à sua carreira, mais relevante é para sua satisfação intrínseca no trabalho que ele esteja desempenhando uma ocupação congruente com seus interesses. Nesse sentido, Dik e Hansen (2011) observaram que quanto menor o envolvimento no trabalho, mais intensa a relação entre congruência e satisfação. Os autores sugeriram que orientadores de carreira devem enfatizar a congruência interesse-ocupação para clientes pouco investidos em seus trabalhos.

Por outro lado, se a carreira é algo muito importante para o trabalhador, ele pode estar satisfeito mesmo em um ambiente incongruente com seus interesses. Nesses casos, a saliência de carreira parece exercer um efeito amortecedor das consequências da incongruência sobre a satisfação. Acredita-se que essas pessoas estejam motivadas e desenvolvam estratégias para modificar ambientes ocupacionais incongruentes, ajustando-os a suas necessidades. Pesquisas futuras de caráter longitudinal seriam úteis para investigar esta possibilidade.

A relação entre congruência e satisfação foi moderada pela diferenciação de interesses, logo, quanto mais diferenciado é o perfil de interesses de um trabalhador, mais importante que ele desempenhe uma ocupação congruente com seus interesses, exceto se seu tipo de interesse predominante for investigativo, para o qual a diferenciação de interesses foi preditor negativo da satisfação intrínseca. Tipos investigativos tendem a ser analíticos, racionais, independentes e inovadores (Holland, 1997). Sua curiosidade natural parece resultar em uma maior consciência e habilidade para especificar interesses, características e objetivos pessoais.
Talvez por isso, tipos investigativos possuam maior identidade vocacional quando comparado aos demais tipos (Leong \& Morris, 1989; Magalhães \& Gomes, 2007). Além disso, tipos investigativos, artísticos e sociais tendem à menor conformidade quando comparados a tipos convencionais, empreendedores e realistas (Hogan \& Blake, 1999). Possivelmente essa menor conformidade contribui para uma maior diferenciação das preferências ocupacionais dos tipos investigativos, artísticos e sociais, uma vez que tendem a não aceitar restrições externas as suas atividades e a acreditar em sua capacidade de desempenhar tarefas à sua própria maneira, geralmente mais inovadora, autônoma e criativa.

Diante disso, recomenda-se que orientadores de carreira estejam atentos aos clientes com inclinações fortemente investigativas, pois a congruência tende a ser mais necessária para sua satisfação no trabalho, uma vez que tais clientes podem vivenciar dificuldades para buscar ou aceitar mudanças de carreira.

Estudos brasileiros sobre os preditores da satisfação no trabalho têm considerado variáveis como características de personalidade, inteligência emocional (e.g., Leite, 2018) entre outras, mas não tem considerado a saliência de carreira como uma dessas variáveis. Esta pesquisa evidenciou que trabalhadores atribuem diferentes níveis de saliência à sua carreira e que sua satisfação com o trabalho depende desse aspecto. Logo, sugere-se que investigações sobre a satisfação com o trabalho considerem o quanto o desenvolvimento da carreira é uma prioridade para os trabalhadores.

É importante destacar que estudos sobre a congruência, referenciados no modelo RIASEC, têm sido criticados por utilizar amostras de estudantes universitários ou por limitar-se a um pequeno grupo de ocupações, o que restringe a variabilidade dos interesses individuais e das demandas ocupacionais, e subestima a relação empírica entre congruência e outras variáveis (Tinsley, 2000). Este estudo buscou superar esses problemas ao investigar trabalhadores das diversas áreas ocupacionais. No entanto, sugere-se que pesquisas futuras preocupem-se com obter uma distribuição equivalente entre os tipos RIASEC, favorecendo comparações e generalizações pertinentes.

Considera-se que, no Brasil, este trabalho inaugura o campo de pesquisas sobre a congruência pessoa-ambiente e seus consequentes. Nesse sentido, uma revisão integrativa das pesquisas nacionais sobre aplicações do modelo de Holland (1997) no contexto organizacional 
e do trabalho pode ser útil para organizar o conhecimento sobre o tema e apontar os próximos passos.

\section{Referências}

Abbad, Gardênia, \& Torres, Cláudio Vaz. (2002). Regressão múltipla stepwise e hierárquica em Psicologia Organizacional: Aplicações, problemas e soluções. Estudos de Psicologia (Natal), 7(spe), 19-29. doi: 10.1590/S1413-294X2002000300004

Allen, S., \& Ortlepp, K. (2002). Conceptualising and operationalising work versus career salience. $S A$ Journal of Industrial Psychology, 28(2), 7-14. doi: 10.4102/sajip.v28i2.45

Amabile, T. M., Hill, K. G., Hennessey, B. A., \& Tighe, F. M. (1994). The Work Preference Inventory: Assessing intrinsic and extrinsic motivational orientations. Journal of Personality and Social Psychology, 66, 950-967. doi: 10.1037/0022-3514.66.5.950

Arnold, J. (2004). The congruence problem in John Holland's theory of vocational decisions. Journal of Occupational and Organizational Psychology, 77, 95-113. doi: 10.1348/096317904322915937

Baron, R. M., \& Kenny, D. A. (1986). The moderatormediator variable distinction in social psychological research: Conceptual, strategic, and statistical considerations. Journal of Personality and Social Psychology, 51, 1173-1182. doi: 10.1037/0022-3514.51.6.1173

Blustein, D. L. (2006). The psychology of working: A new perspective for career development, counseling, and public policy. Mahwah, NJ: Lawrence Erlbaum. doi: 10.1093/oxfordhb/9780199758791.013.0001

Brito, F. S., \& Magalhães, M. O. (2017) Avaliação de ambientes ocupacionais: Construção do Inventário de Classificação Ocupacional. Rev. bras. orientac. prof [online]. 2017, vol.18, n.1, pp. 93-104. ISSN 1984-7270. doi: 10.26707/1984-7270/2017v18n $1 \mathrm{p} 93$

Cavallini, M. (2018). Mulheres ganham menos que os homens em todos os cargos e áreas, diz pesquisa. Recuperado de https://g1.globo.com/ economia/concursos-e-emprego/noticia/ mulheres-ganham-menos-que-os-homens-em-todos-os-cargos-e-areas-diz-pesquisa.ghtml

Dawis, R. V. (2005). The Minnesota theory of work adjustment. Em S. D. Brown \& R. W. Lent (Eds.),
Career development and counseling: putting theory and research to work. (pp. 3-23). New York, NY: Wiley.

Diamond, E. E. (1988). Women's occupational plans and decisions: An introduction. Applied Psychology: An International Review, 37(2), 97-102. doi: 10.1111/j.1464-0597.1988.tb01129.x

Dik, B. J., \& Hansen, J. C. (2011). Moderation of P-E Fit - job satisfaction relations. Journal of Career Assessment, 19, 21-34. doi: 10.1177/1069072710382613

Donohue, R. (2006). Person-environment congruence in relation to career change and career persistence. Journal of Vocational Behavior, 68(3), 504-515. doi: 10.1016/j.jvb.2005.11.002

Greenhaus, J. H. (1971). An investigation of the role of career salience in vocational behaviour. Journal of Vocational Behaviour, 1, 209-216. doi: 10.1016/0001-8791(71)90022-4

Hayes, A. F. (2012-2018). PROCESS for SPSS and SAS (3.1) [software]. Recuperado de http://processmacro.org

Herzberg, F. (1966). Work and the nature of man. Cleveland, $\mathrm{OH}$ : World Publishing Company

Hogan, R., \& Blake, R. (1999). John Holland's Vocational Typology and Personality Theory. Journal of Vocational Psychology, 55, 41-56. doi: 10.1006/ jvbe.1999.1696

Holland, J. L. (1997). Making vocational choices: A theory of vocational personalities and work environments. Odessa, FL: PAR.

Iliescu, D., Ispas, D., Sulea, C., \& Ilie, A. (2014). Vocational fit and counterproductive work behaviors: A self-regulation perspective. Journal of Applied Psychology, Vol 100(1), 21-39. doi: 10.1037/a0036652

Illfelder, J. K. (1980). Fear of success, sex role attitudes, and career salience and anxiety levels of college students. Journal of Vocational Behaviour, 16, 7-17. doi: 10.1016/0001-8791(80)90033-0

Ishitani, T. T. (2010). Exploring the effects of congruence and Holland's personality codes on job satisfaction: An application of hierarchical linear modeling techniques. Journal of Vocational Behavior, 76(1), 16-24. doi: 10.1016/j.jvb.2009.06.014

Kristof-Brown, A. L., \& Billsberry, J. (2013) Fit for the Future, in Organizational Fit: Key Issues and New Directions (eds. A. L. Kristof-Brown and J. Billsberry), 
John Wiley \& Sons, Ltd, Chichester, UK. doi: 10.1002/9781118320853.ch1

Leite, G. A. F., (2018) Infuência da inteligência emocional e personalidade na satisfação professional (Dissertação de mestrado em Gestão de Empresas/MBA). Ponta Delgada: Universidade dos Açores. Recuperado de http://hdl.handle.net/10400.3/4679

Leong, F. T. L., \& Morris, J. (1989). Assessing the construct validity of Holland, Daiger, and Power's measure of vocational identity. Measurement and Evaluation in Counseling and Development, 22, 117-125 doi: 10.1080/07481756.1989.12022920

Magalhães, M. O., \& Gomes, W. B. (2007). Personalidades vocacionais e processos de carreira na vida adulta. Psicologia em Estudo, 12(1), 95-103. ISSN 1413-7372. doi: 10.1590/S1413-73722007000100012

Martins, M. C. F., \& Santos, G. E. (2006). Adaptação e validação de construto da Escala de Satisfação no Trabalho. Psico-USF, 11(2), 195-205. doi: 10.1590/ S1413-82712006000200008

Meir, E. I., \& Segal-Halevi, A. (2001). Environmental congruence, group importance, and well-being among paratroopers. Journal of Career Assessment, 9, 169-183. doi: 10.1177/106907270100900205

Moya, M., Expósito, F., \& Ruiz, J. (2000). Close relationships, gender, and career salience. Sex Roles, 42(9-10), 825-846. doi: 10.1023/A:1007094232453

Nauta MM. (2010). The development, evolution, and status of Holland's theory of vocational personalities: Reflections and future directions of counseling psychology. Journal of Counseling Psychology, 57(1), 11-22. doi: 10.1037/a0028213

Nye, C. D., Su, R., Rounds, J., \& Drasgow, F. (2012). Vocational Interests and performance: A quantitative summary of over 60 years of research. Perspectives on Psychological Science, 7, 384-403. doi: 10.1177/1745691612449021

Pseekos, A. C., Bullock-Yowell, E., \& Dahlen, E. R. (2011). Examining holland's person-environment fit, workplace aggression, interpersonal conflict at work, and job satisfaction: A replication and extension study. Journal of Employment Counseling, 48(2). 63-71. doi: 10.1002/j.2161-1920.2011.tb00115.x

Quinn, R. P., \& Staines, G. L. (1979). Quality of employment survey, 1977: Cross-section. (Institute for Social
Research, University of Michigan). doi: 10.3886/ ICPSR07689.v1

Rolim, L. N. (2018). A inserção da mulher no mercado de trabalho brasileiro. Recuperado de https:/ /www. cartacapital.com.br/blogs/brasil-debate/a-insercao-da-mulher-no-mercado-de-trabalho-brasileiro

Rottinghaus, P. J., Hees, C. K., \& Conrath, J. A. (2009). Enhancing job satisfaction perspectives: Combining Holland themes and basic interests. Journal of Vocational Behavior, 75, 139-151. doi: 10.1016/j. jvb.2009.05.010

Spokane, A. R., Meir, E. I., \& Catalano, M. (2000). Person-environment congruence and Holland's theory. Journal of Vocational Behavior, 57, 137-187. doi: /10.1006/jvbe.2000.1771

Su, R., Murdock, C. D., \& Rounds, J. (2015). Person-Environment fit. Em P. J. Hartung, M. L. Savickas \& W. B. Walsh (Eds.), APA Handbook of Career Interventions (pp. 81-98). Washington, DC: American Psychological Association. doi: 10.1037/14438-005

Teixeira, M. A. P., Castro, G. D., \& Cavalheiro, C. V. (2008). Escalas de Interesses Vocacionais (EIV): Construção, validade fatorial e consistência interna. Psicologia em Estudo, 13(1), 179-186. ISSN 14137372. doi: 10.1590/S1413-73722008000100021

Tinsley, H. E. A. (2000). The congruence myth: An analysis of the efficacy of the person environment fit model. Journal of Vocational Behavior, 56(2), 147-179. Recuperado de http://search.proquest. com $/$ docview $/ 619501730$ ?accountid $=14553$. doi: 10.1006/jvbe.1999.1727

Tracey, T. J. G. (2007). Moderators of the interest congruence-occupational outcome relation. International Journal of Educational and Vocational Guidance, 7, 37-45. doi: 10.1007/s10775-006-9111-3

Tracey, T. J. G., Allen, J., \& Robbins, S. B. (2012). Moderation of the relation between person-environment congruence and academic success: Environmental constraint, personal flexibility and method. Journal of Vocational Behavior, 80(1), 38-49. doi: 10.1016/j. jvb.2011.03.005

Tracey, T. J., Wille, B., Durr, M. R., \& De Fruyt, F. (2014). An enhanced examination of Holland's consistency and differentiation hypotheses. Journal of 
Vocational Behavior, 84(3), 237-247. doi: 10.1016/j. jvb.2014.01.008

Tranberg, M., Slane, S., \& Ekeberg, S. E. (1993). The relation between interest congruence and satisfaction: A meta-analysis. Journal of Vocational Behavior, 42, 253-264. doi: 10.1006/jvbe.1993.1018

Tsabari, O., Tziner, A., \& Meir, E. (2005). Updated meta-analysis on the relationship between congruence and satisfaction. Journal of Career Assessment, 13(2), 216-232. doi: 10.1177/1069072704273165

Upperman, P., J., \& Church, A. T. (1995). Investigating Holland's typological theory with Army occupational specialities. Journal of Vocational Behavior, 47, 61-75. doi: 10.1006/jvbe.1995.1029

Vieira, V. A. \& Faia, V. S. (2014). Efeitos moderadores duplos e triplos na análise de regressão. XXVIII Encontro da Anpad, RJ. Recuperado de http://www. anpad.org.br/admin/pdf/2014_EnANPAD_ EPQ1584.pdf
Wille, B., Tracey, T. J. G., Feys, M., \& De Fruyt, F. (2014). A longitudinal and multi-method examination of interest-occupation congruence within and across time. Journal of Vocational Behavior, 84, 59-73. doi: 10.1016/j.jvb.2013.12.001

Worthington, R. L., Flores, L. Y., \& Navarro, R. L. (2006). Career development in context: Research with people of color. Em S. D. Brown \& R. L. Lent (Eds.), Career development and counseling (pp. 225-252). New York, NY: John Wiley.

Young, G., Tokar, D. M., \& Subich, L. M. (1998). Congruence revisited: Do 11 indices differentially predict job satisfaction and is the relation moderated by person and situation variables? Journal of Vocational Behavior, 52, 208-223. doi: 10.1006/ jvbe.1997.1587

Recebido em: 23/01/2018

Reformulado em: $12 / 08 / 2018$

Aprovado em: 29/08/2018

Sobre os autores:

Fernanda de Souza Brito é graduada, mestre e doutora em Psicologia pela Universidade Federal da Bahia (UFBA) e especialista em Terapia Cognitiva-Comportamental pelo Centro de Psicoterapia Cognitivo Comportamental Wainer \& Piccoloto (WP). Tem, ainda, interesses de pesquisa relacionados à Psicologia das Carreiras (Ajustamento ocupacional, Aposentadoria), Avaliação Psicológica e Desenvolvimento de Instrumentos Psicológicos.

ORCID: 0000-0003-4653-8116

E-mail: fernandadesouzabrito@gmail.com

Mauro de Oliveira Magalhães é doutor em Psicologia pela Universidade Federal do Rio Grande do Sul (2005), professor associado no Instituto de Psicologia da UFBA, membro permanente do Núcleo de Pós-graduação em Administração (NPGA - UFBA), pesquisador e consultor dedicado a psicologia das carreiras, desenvolvimento de lideranças e gestão de pessoas. Realizou, ainda, estágio pós-doutoral na Universidade de Barcelona (Espanha) (2012-2013).

ORCID: 0000-0002-3623-922X

E-mail: maomagal@gmail.com

Contato com os autores:

Instituto de Psicologia da UFBA

Rua Aristides Novis, 197, Estrada de São Lázaro

Salvador-BA, Brasil

CEP: 40210-730

Telefones: 3283-6437 / 3283-6433

Psico-USF, Bragança Paulista, v. 24, n. 2, p. 219-231, abr./jun. 2019 
